\title{
Synergistic Effects of Acetyl-L-Carnitine and Adipose-Derived Stromal Cells on Improving Regenerative Capacity of Acellular Nerve Allograft in Sciatic Nerve Defect
}

\author{
Ghayour Mohammad-Bagher, Abdolmaleki Arash, Behnam-Rassouli Morteza, \\ Mahdavi-Shahri Naser, and Moghimi Ali \\ Department of Biology, Faculty of Science, Ferdowsi University of Mashhad, Mashhad, Iran (G.M.-B., B.-R.M., M.-S.N., M.A.); \\ Department of Engineering Sciences, Faculty of Advanced Technologies, University of Mohaghegh Ardabili, Namin, Iran (A.A.); \\ and Bio Science and Biotechnology Research center (BBRC), Sabalan University of Advanced Technologies (SUAT), Namin, \\ $\operatorname{Iran}(A . A$.
}

Received October 21, 2018; accepted December 19, 2018

\begin{abstract}
The combination of decellularized nerve allograft and adiposederived stromal cells (ASCs) represents a good alternative to nerve autograft for bridging peripheral nerve defects by providing physical guidance and biologic cues. However, the regeneration outcome of acellular nerve allograft (ANA) is often inferior to autograft. Therefore, we hypothesized that acetyl-Lcarnitine (ALCAR) treatment and implantation of ASC-embedded ANA would work synergistically to promote nerve regeneration. Seventy rats were randomly allocated into seven experimental groups $(n=10)$, including the healthy control group, sham surgery group, autograft group, ANA group, ANA + ASCs group, ANA + ALCAR group (50 mg/kg for 2 weeks), and ANA + ASCs + ALCAR (50 mg/kg for 2 weeks) group. All grafts were implanted
\end{abstract}

to bridge long-gap (10-mm) sciatic nerve defects. Functional, electrophysiological, and morphologic analysis was conducted during the experimental period. We found that ALCAR potentiated the survival and retention of transplanted ASCs and upregulated the expression of neurotrophic factor mRNAs in transplanted grafts. Sixteen weeks following implantation in the rat, the ANA supplemented by ASCs was capable of supporting reinnervation across a 10-mm sciatic nerve gap, with results close to that of the autografts in terms of functional, electrophysiological, and histologic assessments. Results demonstrated that ALCAR treatment improved regenerative effects of ANA combined with ASCs on reconstruction of a 10-mm sciatic nerve defect in rat comparable to those of autograft.

\section{Introduction}

Peripheral nerve injury is a common disorder in the clinic. Direct nerve coaptation is the preferred method for repair of nerve transection in the absence of nerve gap. But, where direct repair is impossible, the current clinical gold standard treatment is the nerve autograft (Siemionow et al., 2010). However, clinical application of autograft is limited by the availability of donor nerves, donor site morbidity, and a size mismatch between the donor nerve and the recipient nerve (Mackinnon and Hudson, 1992; Neubauer et al., 2010). This has led to the development of alternatives to autologous nerve grafts. The combination of decellularized nerve allograft and stem cells represents a good alternative to nerve autograft that could be used in the clinic. Acellular nerve allograft preserves a nerve's internal structure, facilitating axonal regeneration. Furthermore, decellularization processes prevent

This work was funded in part by Research Deputy of Ferdowsi University of Mashhad.

https://doi.org/10.1124/jpet.118.254540. immunologic rejection by removing antigenic cellular components. However, regeneration outcome of acellular nerve allograft is often inferior to autograft, perhaps owing to the lack of cellular components, especially Schwann cells, an essential component for peripheral nerve regeneration. They are involved in releasing growth factors and remyelinating regenerated axons (Jessen and Mirsky, 2008). It seems that employment of acellular allograft nerve seeded with Schwann cells is an effective method to repair peripheral nerve defects (Doolabh et al., 1996). However, the difficulties in harvesting and then expanding Schwann cells in vitro for subsequent autologous transplantation and also the morbidity of the donor site strongly limit their use in regenerative medicine (Tohill and Terenghi, 2004; Kingham et al., 2007). Therefore, Schwann cell-like cells derived from stem cells are an attractive alternative because they can be obtained from the patient for use in an autologous therapy (Brohlin et al., 2009).

Autologous cells are generally considered to be more readily accepted by the patient because they do not provoke an immune reaction (Mosahebi et al., 2002). In this regard, adipose-derived stem cells (ASCs) are an easily accessible

ABBREVIATIONS: ALCAR, acetyl-L-carnitine; ANA, acellular nerve allograft; ASC, adipose-derived stromal cell; BDNF, brain-derived neurotrophic factor; CMAP, compound muscle action potentials; DMEM, Dulbecco's modified Eagle's medium; FBS, fetal bovine serum; GDNF, glial cell-derived neurotrophic factor; MSC, mesenchymal stem/stromal cells; MTT, 3-(4,5-dimethylthiazol-2-yl)-2,5-diphenyltetrazolium; NGF, nerve growth factor; PBS, phosphate-buffered saline; SEM, scanning electron microscopy; SFI, sciatic functional index; WRL, withdrawal reflex latency. 
source of adult stem cells that have interest as candidates for autologous cell transplantation (McKenzie et al., 2006; Kuroda et al., 2010; di Summa et al., 2011). The adipose tissue is a mesodermally derived complex tissue that contains, in addition to adipocytes, a stromal population that includes nonadipocyte cells such as stem cells (Kokai et al., 2005). According to the nomenclature established by the International Fat Applied Technology Society, ASCs are adiposederived mesenchymal stem/stromal cells (MSCs) that are adherent in plastic culture dishes (Gimble et al., 2007). The ability of ASCs to differentiate into the glial cell line make them excellent candidates for use as an alternative to Schwann cells for peripheral nerve repair (Kingham et al., 2007; Locke et al., 2009). These properties of ASCs make them attractive cell sources for tissue engineering and regenerative medicine (Gimble et al., 2007). However, the low survival rate and retention of transplanted ASCs limits their therapeutic potential (Potier et al., 2007). It has been shown that most of the transplanted ASCs are lost in the harmful microenvironment of injured tissues, where oxidative stress, energy failure, growth factor deprivation, and inflammatory response can lead to apoptosis (Toma et al., 2002). Therefore, any approach that enhances survival and retention capacity of ASCs may improve the nerve regeneration process and be consequential to functional recovery. In this regard, acetyl-L-carnitine (ALCAR, $\mathrm{C}_{9} \mathrm{H}_{17} \mathrm{NO}_{4}$ ) is an acetylated carnitine [3-hydroxy-4(trimethylammonio)butanoate] derivative with antiapoptotic and antioxidant properties (Tesco et al., 1992; Mansour, 2006). It also shows protective effects in the presence of traumatic neural injuries (Fernandez et al., 1989; Hart et al., 2002). ALCAR interacts with the mitochondrial membrane and plays an important role in the mitochondrial oxidation of fatty acids and the production of cellular energy (Lu et al., 2015). It seems that ALCAR can reduce cytosolic levels of cytochrome $\mathrm{C}$ and subsequent caspase-3 activation (Di Cesare Mannelli et al., 2007). We hypothesized that ALCAR treatment and transplantation of ASCs into a decellularized nerve allograft would work synergistically to promote nerve regeneration. Therefore, the present study was conducted to evaluate the effect of coapplication of systemic administration of ALCAR and ASC-supplemented nerve allograft on bridging a $10-\mathrm{mm}$ gap in the sciatic nerve.

\section{Materials and Methods}

Animals. All experimental procedures were conducted in accordance with the European Union Council Directive of November 24, 1986, and the guidelines of the Ethics Committee of the Ferdowsi University of Mashhad (Iran). All experiments were performed on adult male Wistar rats $(200-250 \mathrm{~g})$. Animals were housed under standard laboratory conditions (temperature, $22 \pm 2^{\circ} \mathrm{C}, 12$-hour light/dark cycle and $60 \% \pm$ $5 \%$ humidity) with free access to food and tap water ad libitum.

Experimental Groups. Seventy rats were randomly allocated into seven groups $(n=10)$, including healthy control, sham surgery, acellular nerve allograft (ANA) without any treatment, ANA seeded with ASCs, ANA treated with ALCAR, ALCAR-treated ASC-ANA construct, and autograft as a positive control group. Two experimental groups were treated daily with ALCAR at a dose of $50 \mathrm{mg} / \mathrm{kg}$ for 2 weeks. ALCAR treatment started immediately after surgery. Regeneration through the ANA treated with ALCAR was compared with regeneration in untreated ANA and autografts. The results of the sham group were similar to the control group; therefore, to avoid giving ineffective information, the results of sham group have not been presented.
Isolation, Culture, and Identification of ASCs. ASCs were harvested from the inguinal fat pad of adult male Wistar rats as previously described (Kingham et al., 2007). In brief, the adipose tissue was enzymatically digested using $0.15 \%(\mathrm{w} / \mathrm{v})$ type I collagenase (Invitrogen/Thermo Fisher Scientific, Waltham, MA) for 1 hour at $37^{\circ} \mathrm{C}$. Enzyme activity was then neutralized by adding phosphatebuffered saline (PBS) supplemented with $1 \%(\mathrm{v} / \mathrm{v})$ fetal bovine serum (FBS). The stromal-vascular fraction (SVF) was then collected by centrifugation at $800 \mathrm{~g}$ for 5 minutes. SVF was then resuspended in low glucose Dulbecco's modified Eagle's medium (DMEM) containing $10 \%(\mathrm{v} / \mathrm{v})$ FBS and $100 \mathrm{IU} / \mathrm{ml}$ penicillin $/ 100 \mu \mathrm{g} / \mathrm{ml}$ streptomycin (Gibco/Thermo Fisher Scientific), and the cells were cultured in $75-\mathrm{cm}^{2}$ flasks (SPL Life Sciences Co., Naechon-myeon Pocheon-si, Gyeonggi-do, Korea) at a density of $1 \times 10^{4} / \mathrm{cm}^{2}$. The cultures were maintained at $37^{\circ} \mathrm{C}$ with $5 \% \mathrm{CO}_{2}$. Cells of passages $3-4$ were used in our experiments. To confirm multipotency, ASCs at passage 3 were cultured in adipogenesis and osteogenesis differentiation media (Invitrogen/Thermo Fisher Scientific) according to manufacturer's protocol. Culture was changed every 3 days. After 3 weeks of culture, ASCs were subjected to Oil Red O staining for adipogenesis, and for osteogenesis they were stained with Alizarin Red for matrix mineralization. To confirm ASC phenotype, $1 \times 10^{5}$ cells $/ \mathrm{ml}$ of cultured cells at passage 3 were subjected to flow cytometry to determine the surface expression of CD11b, CD29, CD31, CD45, and CD90 markers (BD Biosciences, Billerica, MA), as described by the manufacturer. Cells stained with FITC-conjugated IgG1 $\kappa$, IgA, and IgM were used as isotype controls. Flow cytometry was performed by BD FACSCalibur flow cytometer (BD Biosciences), and analysis was performed using FlowJo v 10 (TreeStar, Ashland, OR) software.

ANA Preparation. Sciatic nerves from male Wistar rats were decellularized as previously described by (Sondell et al., 1998). In brief, bilateral sciatic nerves were harvested and trimmed to $15 \mathrm{~mm}$ under aseptic conditions. Nerve tissues were then agitated in deionized distilled water for 7 hours. The nerve tissues were then exposed to $3 \%$ Triton X-100 (Sigma-Aldrich, Milan, Italy) in distilled water for 12 hours, followed by a 24-hour agitation in a solution of $4 \%$ sodium deoxycholate (Sigma-Aldrich) in distilled water. These steps were repeated and, after a final wash with distilled water, acellular nerve segments were stored in PBS (pH 7.4) containing $100 \mu \mathrm{g} / \mathrm{ml}$ penicillin and $100 \mu \mathrm{g} / \mathrm{ml}$ streptomycin at $4^{\circ} \mathrm{C}$ until use. The ends of the tissue were trimmed immediately prior to implantation to attain a clean-cut, 10-mm graft. Hematoxylin and eosin staining and scanning electron microscopy (SEM) were used to observe the histology and ultrastructure of the ANA, respectively.

Histologic Evaluation of ANA. ANA and intact nerve segment were stained with Harris hematoxylin and eosin for observation of general morphology. Briefly, tissue samples fixed in $10 \%$ formalin were paraffin-embedded, and transverse sections of 5 - $\mu \mathrm{m}$ were prepared with a microtome. Tissue sections were deparaffinized by xylene and rehydrated with graded alcohols to $50 \%$ ethanol. After staining with Harris hematoxylin and eosin according to standard procedures, the samples were dehydrated with graded alcohols, cleared in xylene, and mounted in balsam. After drying, the sections mounted in Canada balsam under a coverslip and the slides were observed under light microscopy (Olympus, Tokyo, Japan). In addition, to confirm myelin and axon elimination, transverse sections of ANA were stained with toluidine blue (Fluka/Honeywell Research Chemicals, Bucharest, Romania), as described previously (Raimondo et al., 2009). Briefly, tissue samples were fixed in $4 \%$ paraformaldehyde and dehydrated with ascending ethanol passages. The specimens were embedded in resin and serial crosssections for each sample were cut at $1-\mu \mathrm{m}$ thickness with an ultramicrotome. For tissue analysis, resin sections were stained with $1 \%$ toluidine blue, and the prepared slides were observed under a light microscopy (Olympus).

DAPI Staining. To confirm the cells' elimination, transverse sections of ANA were stained with DAPI as described previously. Briefly, tissue sections were deparaffinized by xylene and rehydrated 
with graded ethanol. The slides were drained and incubated with DAPI staining solution $(200 \mu \mathrm{l})$ for 15 minutes in dark. The slides were mounted with Entellan (MilliporeSigma, St. Louis, MO) and observed under fluorescence microscope.

Tensile Testing of ANA. ANA were loaded onto a Universal Testing Machine (SANTAM-STM20; Tehran, Iran). Specimens were attached to the tensile tester. Each specimen was stretched at a constant rate of $0.1 \mathrm{~mm} / \mathrm{s}$ to complete tensile failure. The mean specimen length ( $n=10$ /group) was $10 \mathrm{~mm}$, and samples were kept moist by applying PBS to the specimens. Normal sciatic nerves were used as controls.

In Vitro Biocompatibility Assay of ANA. Briefly, $5 \times 10^{5} \mathrm{ASCs}$ were seeded onto each of the prepared ANA (5 mm) under a stereomicroscope (Nikon, Tokyo, Japan). The cell-ANA combinations allowed to attach at $37^{\circ} \mathrm{C}$ for 4 hours. The cell-ANA combinations were then placed in six-well plates containing DMEM with $10 \%$ fetal bovine serum for 24 hours at $37^{\circ} \mathrm{C}$ and $5 \% \mathrm{CO}_{2}$ under saturated humidity. After the constructs were cultured for 24 hours, cell attachment was evaluated by S.E.M. micrographs. To evaluate ANA biocompatibility, the viability and proliferation of ASCs that seeded on ANA were compared with ASCs cultured in 96 -well plates. Briefly, $10 \mu \mathrm{l}$ of ASCs suspension with a density of $3 \times 10^{5} / \mathrm{ml}$ was seeded onto the 5 -mm ANA and allowed attachment for 4 hours. The cell-ANA combinations were incubated on 96-well plates containing DMEM with $10 \% \mathrm{FBS}$ at $37^{\circ} \mathrm{C}$ with $5 \% \mathrm{CO}_{2}$ for 96 hours. In the control group, ASCs were cultured in 96 -well plates at the same density. MTT assay was performed every 24 hours. Briefly, samples were incubated with $20 \mu \mathrm{l}$ of MTT reagent $(5 \mathrm{mg} / \mathrm{ml})$ in the dark at $37^{\circ} \mathrm{C}$ for 4 hours. After the addition of $200 \mu \mathrm{l}$ of dimethyl sulfoxide (MilliporeSigma) for 1 hour, the absorbance of colored formazan products was measured at a wavelength of $570 \mathrm{~nm}$ by a microplate reader (Model 550; Bio-Rad Laboratories, Hercules, CA). Absorbance values correspond to the number of viable cells. Tissue culture polystyrene and untreated ANA were used as controls.

Surgical Procedure and Implantation. Before implantation, $1 \times 10^{6}$ ASCs were resuspended in $20 \mu \mathrm{l}$ of DMEM and injected into the ANA $(10 \mathrm{~mm})$. The cell-ANA combination was incubated for 4 hours at $37^{\circ} \mathrm{C}$ with $5 \% \mathrm{CO}_{2}$ to allow cells to attach to the ANA. ASC-ANA constructs were pretreated with acetyl-L-carnitine $(10 \mathrm{mmol})$ for 12 hours until surgical implantation. All experiments were performed under an operating microscope (Zeiss, Berlin, Germany) under sterile conditions by the same investigator. All animals were deeply anesthetized using an intraperitoneal injection of ketamine $(80 \mathrm{mg} / \mathrm{kg})$ and xylazine $(10 \mathrm{mg} / \mathrm{kg}$ ) (Alfasan Pharmaceutical Co., Woerden, The Netherlands). Animals were fixed in the prone position and the left sciatic nerve was exposed through a longitudinal incision extending from the greater trochanter to the mid-thigh. Then, the nerve was transected and a $6 \mathrm{~mm}$-long segment of the sciatic nerve was removed at $1 \mathrm{~cm}$ below the sciatic notch. Then, a $10 \mathrm{~mm}$ prepared ANA was grafted to the nerve defect and fixed with four epineural microsutures (10-0 nylon; Ethicon, Somerville, NJ). For autografts, $10 \mathrm{~mm}$ of the sciatic nerve was transected, reversed $180^{\circ}$, and then reimplanted into the nerve gap. Finally, the muscle and fascia were sutured with absorbable sutures (4-0 Vicryl; Ethicon) and the skin by a continuous running suture (4-0 Prolene; Ethicon). The sham-operated group was subjected to the surgical procedure without the nerve defect. During the study, animals were examined for signs of autotomy and contracture. Immediately after surgery, animals in ALCAR treatment groups were intraperitoneally injected with ALCAR (MilliporeSigma; $50 \mathrm{mg} / \mathrm{kg}$ per day) for 14 days.

Functional Assessment. The recovery of motor function was assessed by calculating the sciatic functional index (SFI) before nerve transection and at $4,8,12$, and 16 weeks postoperative, as previously described (Dijkstra et al., 2000). The SFI value was calculated by putting the obtained data into the formula: SFI $=-38.3[(\mathrm{EPL}-\mathrm{NPL}) /$ NPL $]+109.5[($ ETS-NTS $) / \mathrm{NTS}]+13.3[($ EIT-NIT $) / \mathrm{NIT}]-8.8$, where EPL is the experimental paw length, NPL is the normal paw length, ETS is the experimental toe spread, NTS is the normal toe spread, EIT is the experimental intermediary toe spread and, NIT is the normal intermediary toe spread. The SFI value varies from 0 to -100 , with 0 corresponding to the normal function and -100 indicating total impairment. When no footprints were measurable, the index score of -100 was given. Sensory recovery was evaluated in the same sessions as the hot-plate test. The hot-plate is the widely used test to assess thermal nociception. In brief, the rat was wrapped in a surgical towel above its waist and then positioned to stand with the affected hind paw on a hot plate at $54^{\circ} \mathrm{C}$ to assess the nociceptive withdrawal reflex latency (WRL), defined as the time elapsed from the onset of hotplate contact to withdrawal of the hind paw (Hargreaves et al., 1988). The cut off time for heat stimulation was set at 12 second, to avoid skin damage to the foot.

Electrophysiological Evaluation. To assess the regeneration process, electrophysiological recordings were conducted at 8 and 16 weeks postoperative. For this purpose, the compound muscle action potentials (CMAPs) peak amplitude and motor nerve conduction velocities (MNCVs) were measured between the injured side and the contralateral uninjured side, as previously described (Navarro and Udina, 2009; Oğuzhanoğlu et al., 2010). In brief, the sciatic nerve was stimulated by a miniature bipolar electrode, which was placed on the nerve proximal to the graft. The active and reference monopolar needle recording-electrodes were inserted into the midbelly and tendon surface of gastrocnemius muscle, respectively. A ground electrode was placed in the surrounding tissue. Stimulations $(1 \mathrm{~Hz}$; $1 \mathrm{~mA}$ ) with durations of 0.02 milliseconds were given at gradually increasing intensity until a maximal CMAP response was obtained. For evaluation of MNCVs, two different points along the proximal nerve segment were stimulated. MNCVs across the segment were determined by calculating the distance between the different stimulating points and the difference in latencies $(\Delta \mathrm{t})$. Normal nerve CMAPs and MNCV were measured from the contralateral uninjured side in a similar fashion.

Histomorphometry Analysis. Following the electrophysiology study, all of the implanted grafts were harvested and subjected to toluidine blue staining for morphometric analysis as described previously. Briefly, transverse semithin sections $(1-\mu \mathrm{m})$ were stained with $1 \%$ toluidine blue. Axon counts, axon diameter, and myelin thickness were calculated using the Image J program. The contralateral sciatic nerves were used as controls.

Wet Gastrocnemius Muscle Weights. Muscle weight was measured to assess denervation atrophy at 16 weeks postoperative as previously described (Ghayour et al., 2017). The gastrocnemius muscles were harvested and the ratio of mass muscle of the operated side to the contralateral side was calculated immediately. The muscle mass ratio represented the recovery in denervation atrophy of the gastrocnemius muscle on the operated side, with approximately $100 \%$ gastrocnemius muscle index indicating full recovery of the operated side.

Quantitative Real-Time Polymerase Chain Reaction. The pattern of gene expression for key markers of the Schwann cell lineage (S100 and p75) and neurotrophins [nerve growth factor (NGF), brainderived neurotrophic factor (BDNF), and glial cell-derived neurotrophic factor (GDNF)] were compared in ASCs seeded on ANA. Also the expression of glyceraldehyde 3-phosphate dehydrogenase (GAPDH) as an internal control was evaluated. Total mRNA was extracted from tissue samples using TRIzol reagent (Invitrogen/Thermo Fisher Scientific). DNase I (Fermentas/Thermo Fisher Scientific) was used to remove DNA contamination, and mRNA was quantified by spectrophotometer (Nano Drop ND-1000; Thermo Fisher Scientific). Total RNA (5 $\mu \mathrm{g}$ of mRNA) was then reverse-transcribed with Moloney murine leukemia virus reverse transcriptase kit (Invitrogen/Thermo Fisher Scientific), and for cDNA synthesis we used PrimeScript RT Reagent Kit (Takara Biotechnology, Dalian, China). The cDNA (200 ng) was amplified in the presence of $2 \mu$ l of Power SYBR Green Master Mix (Applied Biosystems/Thermo Fisher Scientific, Foster City, CA) and the appropriate primers at $250 \mathrm{nM}$ concentration using a real-time polymerase chain reaction (PCR) system (40 cycles; Applied Biosystems 
StepOne Real-Time PCR System (StepOne system)) and analyzed with the StepOne Software (Applied Biosystems/Thermo Fisher Scientific). Analyses were carried out using the $\Delta \mathrm{Ct}$ method and calculated relative to GAPDH. The results were normalized with respect to the control condition, which presented a value of 1 . The primers were synthesized based on information from GenBank as follows: NGF, forward 5'GCCCACTGGACTAAACTTCAGC-3', reverse 5' -CCGTGGCTGTGGTCTTATCTC-3'; BDNF, forward 5'-GGTCACAGTCCTGGAGAAAG-3', reverse 5'-GTCTATCCTTATGAACCGCC-3'; GDNF, forward 5'-GGATGTCGTGGCTGTCTG-3', reverse 5'-GCCGCTTGTTTATCTGGT-3'; S-100, forward 5-GTCCACACCCAGTCCTCTCTGGAG-3', reverse 5'CCGGAGGCTCCTGGTCACCTTTTG-3'; P75, forward 5'-GGGCTGATGCTGAATGCGAAGAGAT-3', reverse 5'-GCCCACGACCACAGCAGCCAAGAT-3'; GAPDH, forward 5'-CCACGGCAAGTTCAACGGCACAGT-3' and reverse 5'-CAGCGGAAGGGGCGGAGATGAT-3' (Hea Gu et al., 2012; Liu et al., 2011). The assay was repeated three times.

Statistical Analysis. Data were analyzed with SPSS Statistics 16.0 software (SPSS Inc., Chicago, IL). Statistical analysis was carried out using a one-way analysis of variance to determine the significant differences among seven groups. Intergroup comparison of means was performed using a Tukey post-hoc analysis.
All data are expressed as mean \pm S.E.M. and values of $P<0.05$ were considered statistically significant.

\section{Results}

ASCs Characterization. Under inverted phase microscopy, cultured ASCs at passage 3 had a spindle-like morphology (Fig. 1B). To confirm ASCs characterization, immunophenotype of cultured ASCs at passage 3 was performed by FACS analysis of surface marker expression. Results showed that ASCs expressed a high level of CD29 $(98.7 \% \pm 1.3 \%)$ and CD90 $(98.6 \% \pm 0.67 \%)$ but were negative for endothelial marker CD31 $(0.45 \% \pm 0.01 \%)$ or the hematopoietic lineage markers CD45 $(2.26 \% \pm 0.3 \%)$ and CD11b $(1.68 \% \pm 0.4 \%)$ (Fig. 1A). These results were in agreement with previous reports. Also, to confirm multipotency capacity, ASCs were cultured in osteogenic and adipogenic media. After culturing the ASCs in osteogenic medium, the mineralization of calcium was detected by Alizarin Red S staining (Fig. 1C).
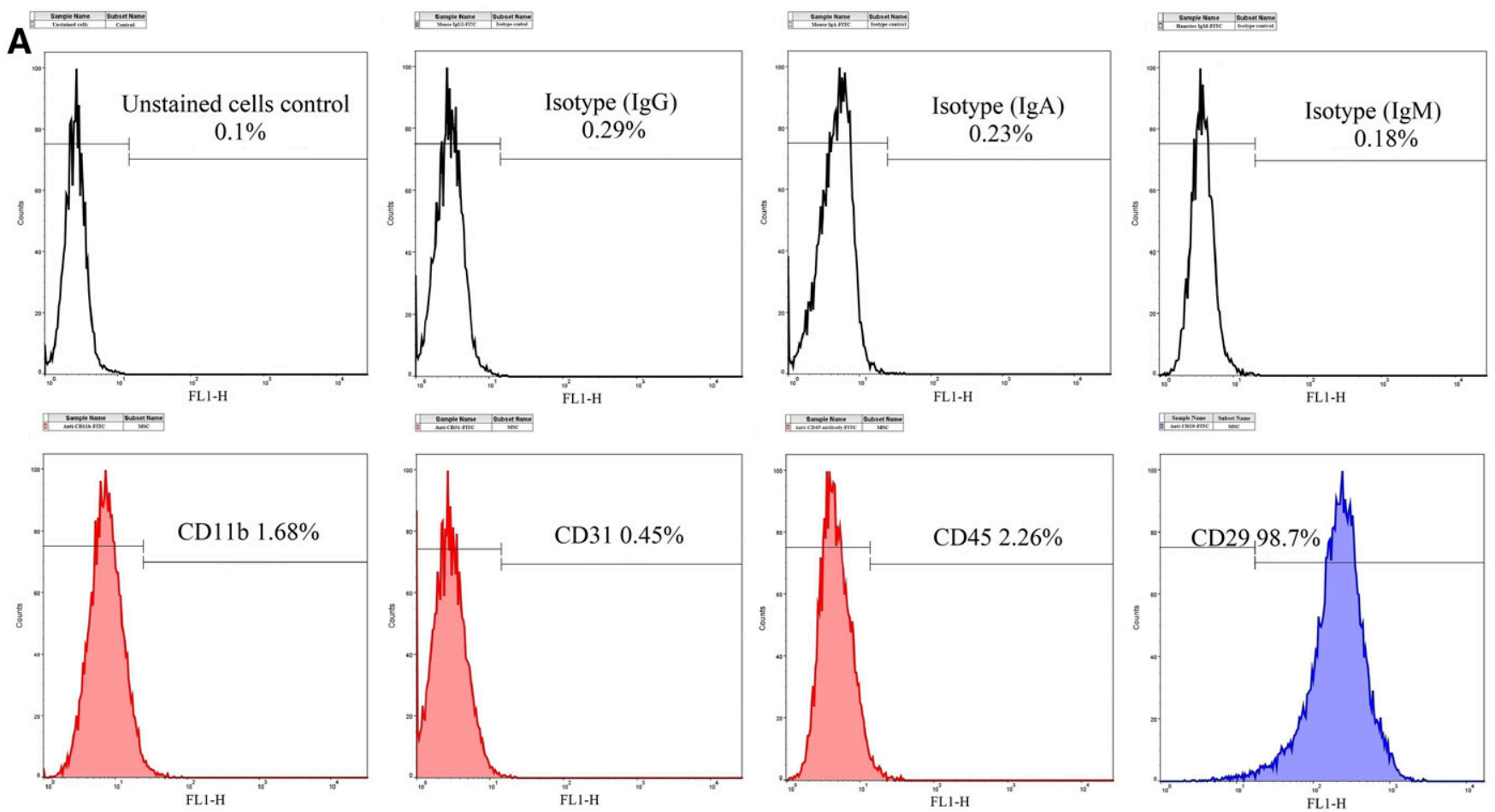

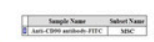



B

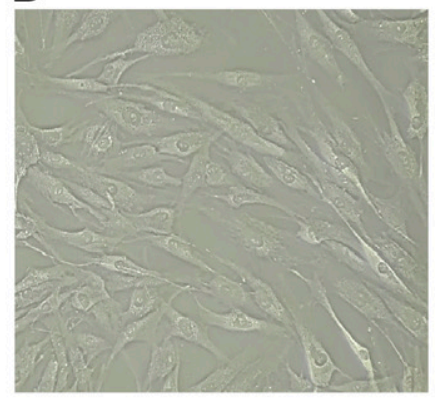

C

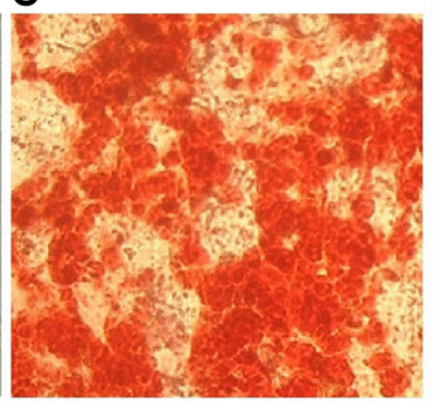

D





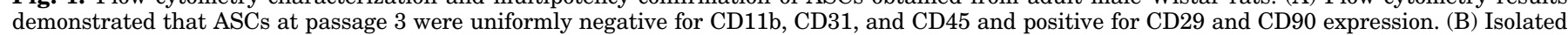





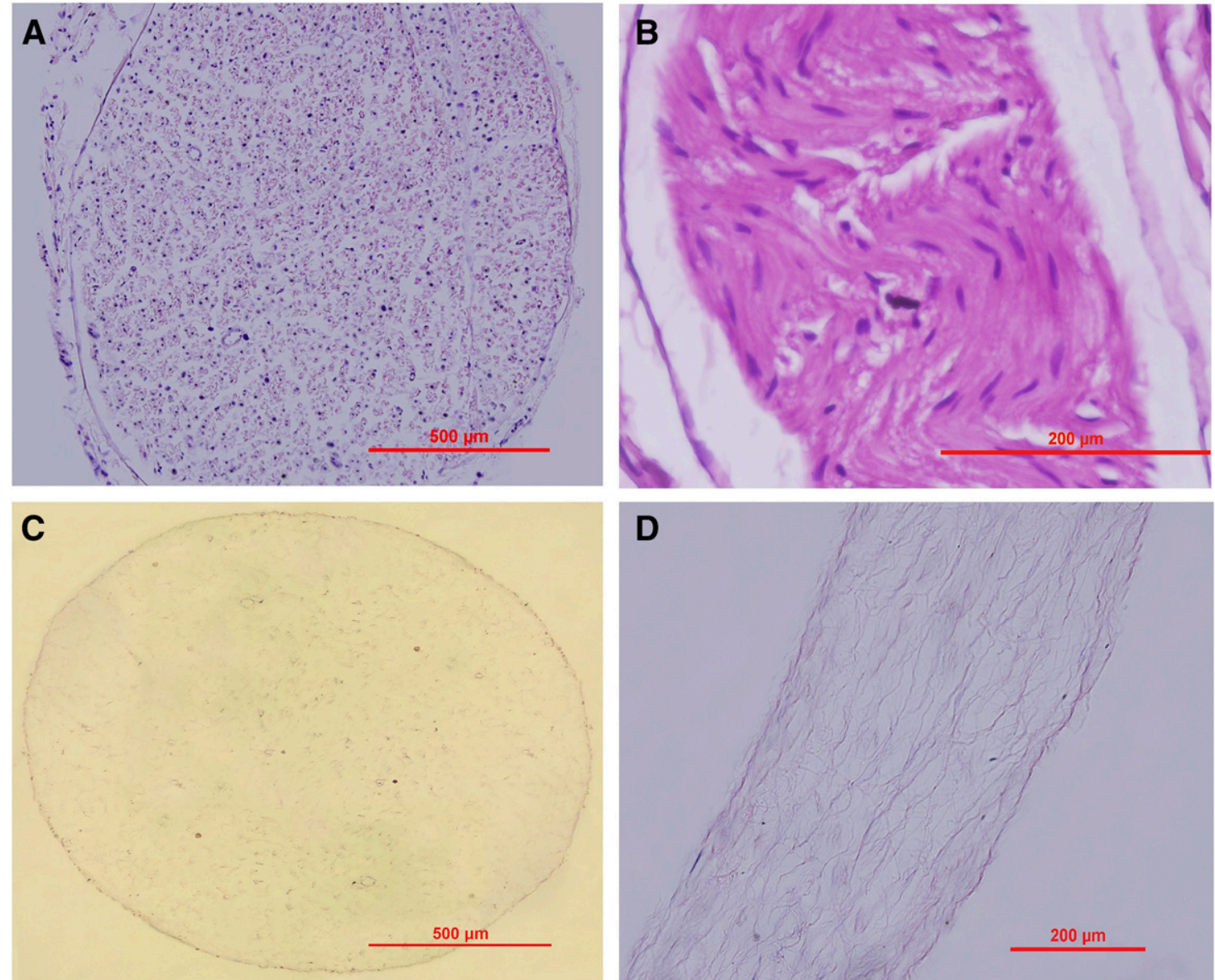

Fig. 2. Hematoxylin and eosin staining was used to compare the general morphology of fresh rat sciatic nerve to acellular nerve allograft (Sondell protocol). (A and C) Cross sections of intact nerve and acellular nerve, respectively. (B and D) Longitudinal sections of intact nerve and acellular nerve, respectively. Schwann cell nuclei were stained in deep blue (arrowhead) and the myelin sheaths were mesh-like with faint staining in fresh nerve segment. Arrows, nerve fibers (axons and myelin sheaths). But, in ANA, axons and cell nuclei have disappeared.

Also, following adipogenic differentiation, the accumulation of intracellular lipid vacuole accumulation was detected by Oil Red O staining (Fig. 1D).

Characterization of ANA. According to the results, the color of prepared ANA was white and transparent. The elasticity of ANA was lower than fresh nerves, and the diameter and length were slightly decreased compared with normal nerves. Hematoxylin and eosin staining (Fig. 2) and DAPI staining (Fig. 3) showed that axon, myelin sheath, and cell nuclei had disappeared in decellularized nerves, and
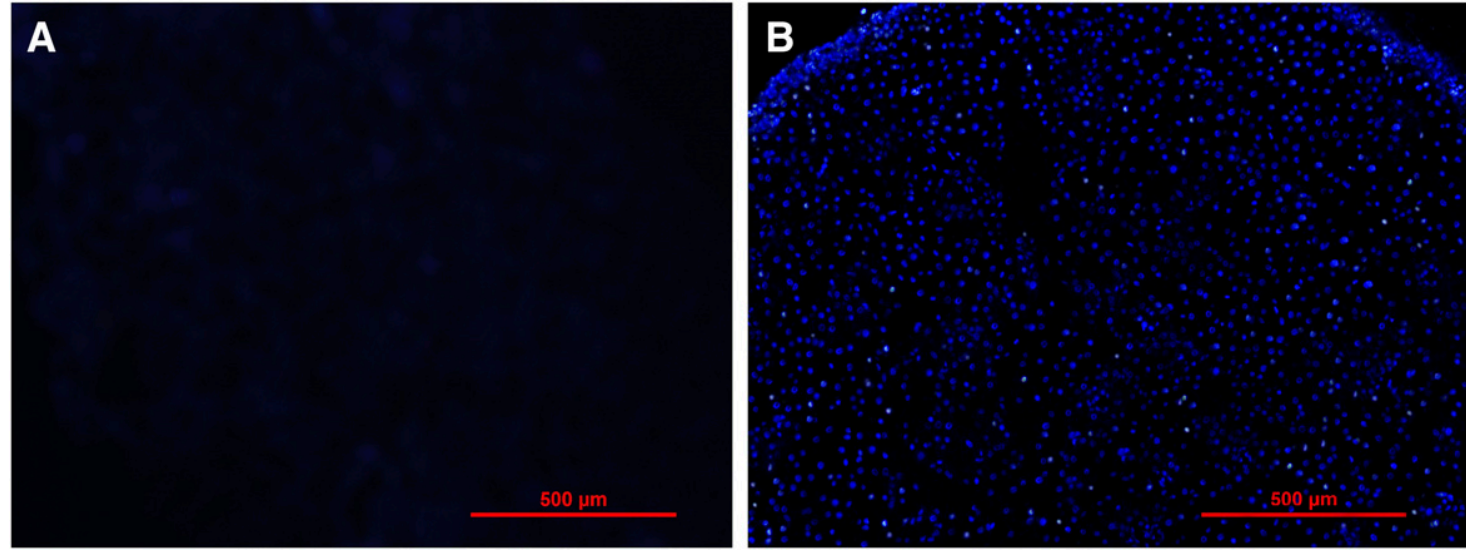

Fig. 3. DAPI-stained cross sections of rat sciatic nerve were used to compare the cell's nucleuses of (A) freshly dissected nerve to (B) decellularized nerve segment. Results showed that the cell nucleuses had disappeared in decellularized nerve. ANA cross sections were stained with DAPI and immediately imaged by fluorescence microscopy. (A) Control segment from freshly dissected nerve. (B) Cross section from nerve segment after decellularization. 



Fig. 4. Toluidine-blue staining was conducted on samples of the acellular nerve to confirm decellularization process. (A and C) Cross sections of fresh nerve and acellular nerve, respectively. (B and D) Longitudinal sections of fresh nerve and acellular nerve, respectively. Myelin sheath and axon have disappeared in the acellular nerve segment.

the basement membrane was arranged in a wave-like manner. Normal nerve exhibited intact axons and the myelin sheath was mesh-like with faint staining. Schwann cells could be seen as deep blue in the normal nerves (Fig. 2, A and B). Also, the removal of myelin was evaluated by staining tissue sections for lipids with toluidine blue. Photographs showed the regular topography of normal nerves and well-myelinated axons of varying diameter (Fig. 4, A and B). However, myelinated axons had disappeared in ANA sections (Fig. 4, C and D). Moreover, SEM revealed that the three-dimensional structures of ANA were preserved at an acceptable level.
Micrographs showed that the morphology of the ANA was partly changed after the chemical decellularization process (Fig. 5, B and C).

Tensile Testing of ANA. Peripheral nerve trunks are viscoelastic tissues with unique mechanical characteristics. Tensile testing was performed to determine the difference in tensile strength between fresh and decellularized peripheral nerve. There were no differences in the average lengths and widths of the specimens. Result showed that the mean ultimate stress, ultimate load, and breaking stress for ANA decreased significantly compared with the averages for intact
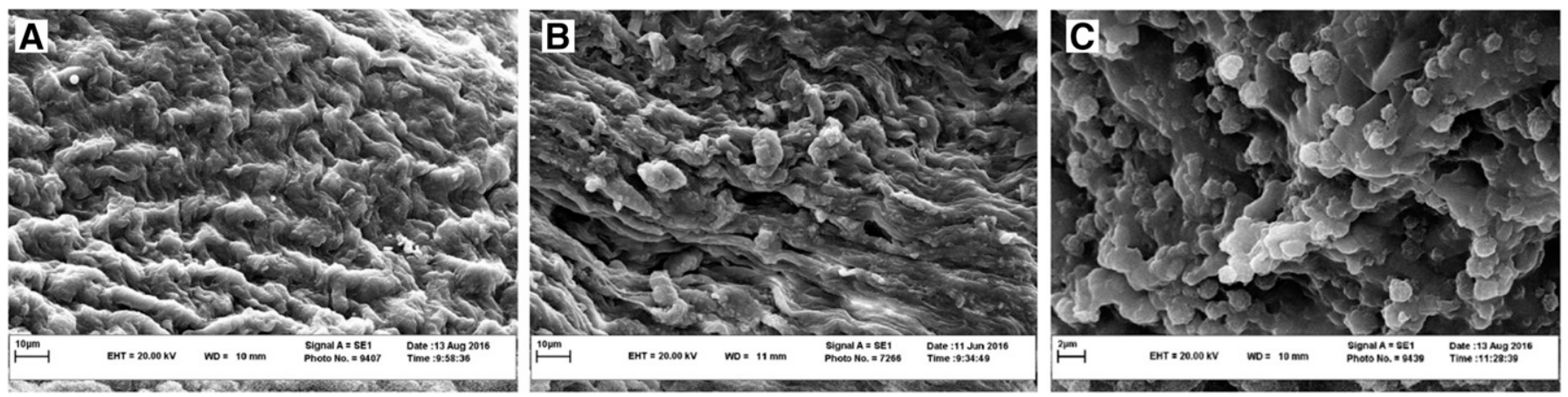

Fig. 5. Scanning electron microscopy of longitudinal nerve and ANA. Longitudinal sections of fresh nerve (A) and acellular nerve (B). The threedimensional structure of acellular nerve was not severely destroyed by decellularization process. After coculture for 4 hours, the cells are adhering to the ANA (C). 
TABLE 1

Tensile testing of acellular nerve and normal sciatic nerves $(n=10)$

\begin{tabular}{lccccc}
\hline Groups & Peak Force & Ultimate Stress & Ultimate Strain & Peak Extension & Break Stress \\
\hline & $N$ & $M P a$ & $\%$ & $m m$ & $M P a$ \\
Control & $2.04 \pm 0.55$ & $0.65 \pm 0.16$ & $42.02 \pm 18.12$ & $4.22 \pm 1.79$ & $0.04 \pm 0.05$ \\
ANA & $1.35 \pm 34^{*}$ & $0.41 \pm 0.11^{*}$ & $64.33 \pm 15.1^{*}$ & $6.3 \pm 1.47^{*}$ & $0.2 \pm 0.15^{*}$ \\
\hline$* P<0.05$. & & & &
\end{tabular}

nerve. Albeit sufficiently strong for reconstructive methodology, the tensile strength of ANA had diminished in comparison with fresh nerve (Table 1).

Biocompatibility. ANA biocompatibility assay was performed for evaluating cell retention capacity of ANA before in vivo implantation. After a 4-hour incubation of ASC-ANA constructs, SEM micrographs showed that cells adhered well to the ANA (Fig. 5, C and D). SEM micrographs showed that ASCs were evenly distributed in the basilar membrane of ANA. After culturing for 96 hours, MTT assay showed that cell viability and proliferation were not significantly different at 24 and 48 hours among ASCs seeded on ANA and ASCs cultured in 96 -well plates. With increasing culture time, cell proliferation rate of the control group was significantly higher than the ASC-ANA construct group at 72 and 96 hours. These results indicated that ANA had no toxic effects on implanted ASCs, after culture for 96 hours. The data are expressed as the ratio of the mean of the optical density of the treated groups to that of the control group $(P<0.05$; Fig. 6$)$.

Functional Evaluation. Behavioral analysis was performed to evaluate motor and sensory function recovery. All animals survived and recovered from anesthesia. No surgical complications occurred, except autotomy of the experimental foot following sciatic nerve axotomy. Autotomy was detected in two animals from the ANA group, one animal from autograft group, and one animal from ANA+ASCs group. Autotomy leads to premature euthanasia of the animals by $\mathrm{CO}_{2}$ inhalation. Also, no side-effects were observed as a result of the 2-week daily intraperitoneal injection of ALCAR. The recovery of locomotive function in rats was evaluated by calculating the SFI. The SFI value varies from 0 to -100 , with 0 corresponding to the normal function and -100 corresponding to complete dysfunction. The mean SFI value in all groups was approximately $(-5 \pm 0.78)$ before surgery, indicating normal function. At 4 weeks postoperative, the SFI values in all experimental groups decreased dramatically to the lowest level with no obvious difference, indicating complete loss of function. At 8,12 , and 16 weeks postoperative, the SFI value in autograft group and ALCAR-treated ASC-ANA construct group showed the greatest significant improvement of all groups $(P<0.05)$. According to the results, the SFI value between autograft group and ALCAR-treated ASC-ANA construct group was not statistically significant at 16 weeks postoperative (Fig. 7). Also, the hot-plate test was carried out to assess the thermal nociceptive threshold in all groups. Figure 8 shows the data for the WRL tests. In the 4th week post-surgery, all animals presented severe loss of sensory function and all tests had to be interrupted at the selected cut-off time of 12 seconds. Nociception recovered significantly in the following weeks, except in the negative control group. The results indicated significant differences between groups in the recovery of nociception. According to the results, the WRL value between the autograft group and ALCAR-treated ANA groups was not statistically significant at 16 weeks postoperative. However, at 16 week postoperative, the WRL value in ALCAR-treated ASC-ANA construct group showed significantly greater recovery than the autograft group $(P<0.05)$.

Electrophysiological Evaluation. Electrophysiological test was performed for quantitative measurement of electrical motor nerve activity and muscle reinnervation. According to the results, the CMAPs amplitude increased and CMAPs onset latencies decreased progressively with time in all groups. MNCV was calculated on the basis of CMAP onset latency, and the distance between the recording and stimulating electrode tips. The results indicated that the ALCARtreated ASC-ANA construct group and autograft group achieved significantly higher CMAP peak amplitude, shorter onset latency, and higher MNCV than other experimental groups at 16 weeks postoperative $(P<0.05$; Fig. 9$)$. However, the CMAP peak amplitude and MNCV for the ALCAR treatment groups were significantly lower than for the sham group $(P<0.05)$. At 16 weeks postoperative, the CMAP amplitude increased and CMAP onset latencies of regenerated nerves in the ALCAR-treated ASC-ANA construct group were similar to that in the autologous nerve group.

Histomorphometry Analysis. Morphometric analysis of regenerated sciatic nerves was performed to quantitatively estimate myelinated nerve fiber number and fiber size parameters. In this regard, toluidine blue-stained semithin transverse sections of distal regenerated nerve stumps were
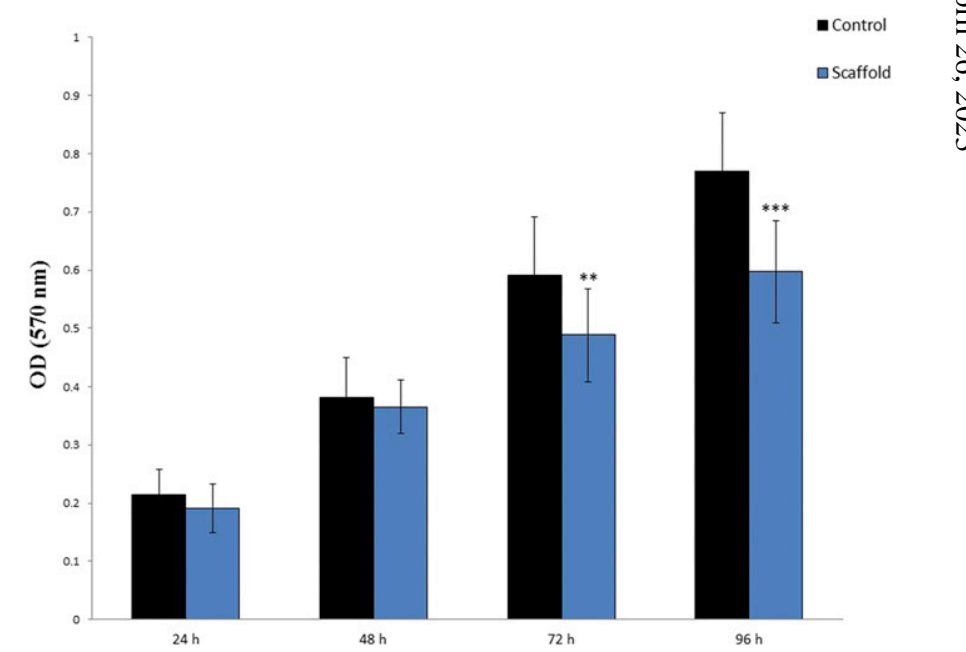

Fig. 6. Evaluation of ANA biocompatibility. The viability and proliferation of ASCs seeded onto ANA was compared using MTT assay with ASCs cultured in 96-well plates. MTT assay shows that ANA had no toxic effect on cell viability and proliferation under normal culture conditions. The experiments were performed in triplicate $(n=10)$. $* * P<0.01$ and $* * * P<0.001$. 


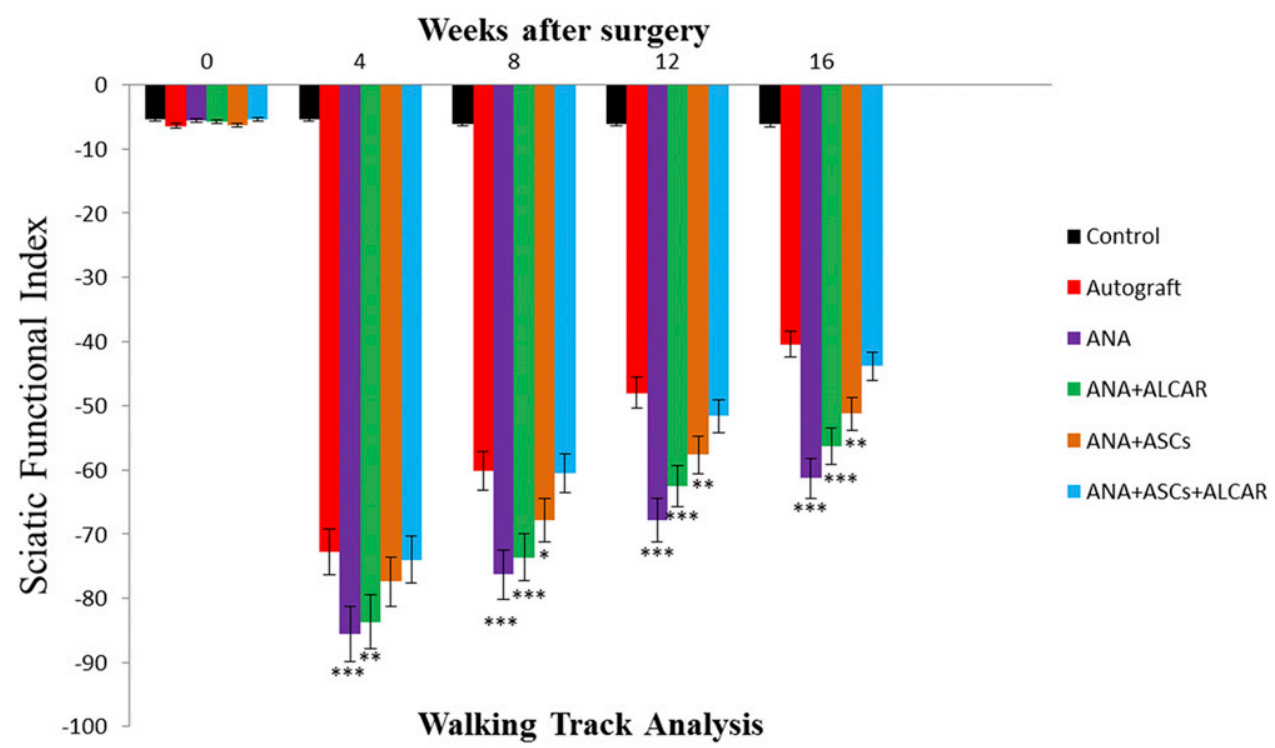

Fig. 7. Sciatic function index was measured every 4 weeks after surgery in each experimental group. Data are expressed as mean \pm S.D. $(n=10) . * P<0.05 ; * * P<0.01$; $* * * P<0.001$ vs. autograft group at the same time point.

examined by optical microscopy (Fig. 10). Table 2 shows quantitative morphometric analyses of regenerated nerves for each of the experimental groups. At the end of 16 weeks postoperative, morphometric parameters, including total myelinated fiber number, myelin thickness, axon diameter, and axonal density, were significantly greater in autograft and ALCAR-treated ASC-ANA construct groups compared with other groups, except control $(P<0.001)$. However, the total myelinated fiber number in the ALCAR-treated ASC-ANA construct group was significantly greater than autograft $(P<0.05)$, but no significant difference was found between the ALCAR-treated ANA group and autograft group. Moreover, myelin thickness in the ALCAR-treated ANA group was significantly greater than ASC-ANA group $(P<0.05)$. But, it was significantly inferior compared with the autograft and ALCAR-treated ASC-ANA construct groups $(P<0.05)$. There were no significant differences between the results of autograft and ALCAR-treated ASC-ANA construct groups at 16 weeks postoperative. Also, the fiber diameter in the autograft and ALCAR-treated ASC-ANA construct groups was significantly greater than other experimental groups $(P<0.05)$, while no other significant difference was found between the two groups.

Muscle Mass and Morphology. Sciatic nerve transection produces the loss of the neural innervation to the gastrocnemius muscle, which leads to a decrease in the muscle mass. Therefore, muscle weight was measured to assess denervation atrophy at the end of experiment. According to results, the

\section{Hot plate test}

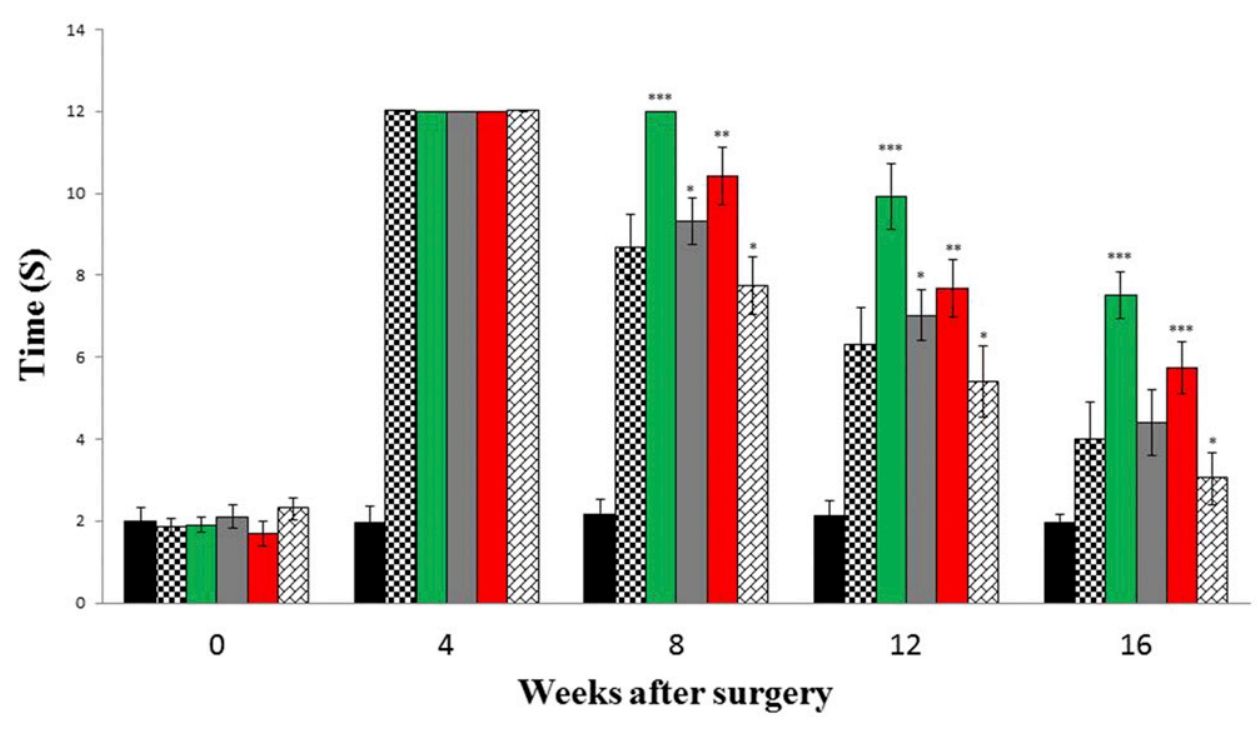

Fig. 8. Withdrawal reflex latency test using a hot plate was performed every 4 weeks after surgery in each experimental group. Data are expressed as mean \pm S.D. $(n=10) . * P<0.05$; $* * P<0.01$; *** $P<0.001$ vs. autograft group at the same time point. 

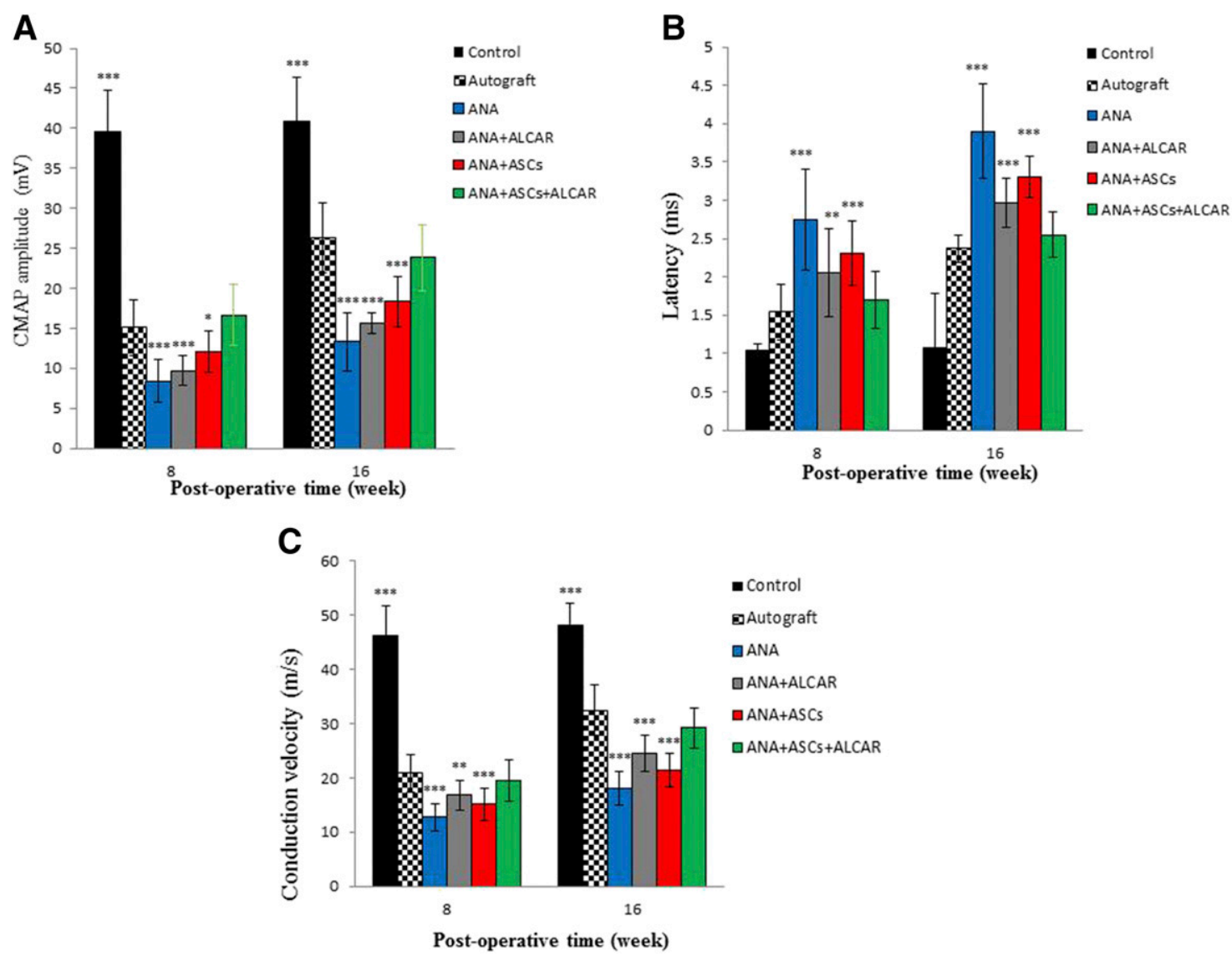

Fig. 9. (A) Representative results of CMAP amplitude measurements after proximal stimulation of operated and unoperated sciatic nerves at 8 and 16 weeks post-surgery. Data are shown as mean \pm S.D. $(n=10)$. $* P<0.05 ; * * * P<0.001$ vs. autograft group. (B) Representative results of CMAP delay measurements at 8 and 16 weeks post-surgery. The data are shown as mean \pm S.D. $(n=10)$. ${ }^{* *} P<0.01 ; * * * P<0.001$ vs. autograft group. (C) Representative results of conduction velocity measurements at 8 and 16 weeks post-surgery. The data are shown as mean \pm S.D. $(n=10)$. $* * P<0.01$; $* * * P<0.001$ vs. autograft group.

experimental gastrocnemius muscles exhibited atrophy compared with the contralateral side in all groups. At 16 weeks after surgery, the muscle weight showed a recovery up to $54.1 \% \pm 0.5 \%$ in the autograft group and $58.3 \% \pm 0.6 \%$ in the ALCAR-treated ASC-ANA construct group. Also, the results showed that muscle mass ratio was higher in the ALCARtreated ANA groups than ASC-ANA construct and ANA groups. These results indicated that gastrocnemius muscle atrophy was reduced by systemic administration of ALCAR. No significant difference was observed between the ALCARtreated ASC-ANA construct and autograft group (Fig. 11).

Quantitative Real-Time PCR. Schwann cells and neurotrophic factors are keys factors in the nerve regeneration process. In this regard, nerve grafts were subjected to quantitative real-time PCR to evaluate the mRNA levels of neurotrophin (NGF, GDNF and BDNF) genes and Schwann cell lineage-related genes (S100 and p75) at 2 weeks postoperative. Results showed that the ALCAR-treated ASC-ANA construct group expressed a wide range of neurotrophin genes. According to the results, NGF, BDNF, and GDNF were significantly upregulated in both the autograft group and ALCAR-treated ASC-ANA construct group $(P<0.01$; Fig. 12). Importantly, there was significant difference between the ALCAR-treated ASC-ANA construct group and ASC-ANA construct in terms of NGF and GDNF gene expression $(P<0.01 ;$ Fig. 12). Also, the pattern of gene expression for key markers of the Schwann cell lineage (S100 and p75) was compared in all groups. Real-time PCR analysis indicated that Schwann cell marker mRNAs were significantly upregulated in the ALCAR-treated ASC-ANA construct group in comparison with the ASC-ANA construct group and ALCAR-treated ANA group $(P<0.001 ;$ Fig. 12). This finding suggests that the number of Schwann cells increased in the ALCAR-treated ASC-ANA construct group. Moreover, our results showed that ALCAR treatment decreased caspase 3 mRNA expression at the site of nerve injury in ANA-ASCs construct-engrafted rat.

\section{Discussion}

Acellular nerve allograft is a good alternative to autograft. However, animal research indicates that the regenerative effect of acellular nerve allograft is still lower than autograft owing to the lack of an appropriate microenvironment for axonal regeneration. It seems that implanted ASCs could improve the microenvironment of ANA through secretion of neurotrophins and growth factors (Zhang et al., 2010). However, transplanted ASCs encounter an ischemic microenvironment characterized by hypoxia and serum and nutrition deprivation (Potier et al., 2007). Metabolic stress is a main cause of the limitedviability of ASCs after transplantation, and the short lifespan of transplanted ASCs limits their therapeutic efficacy (Potier et al., 2007; Chavakis et al., 2008). It is 

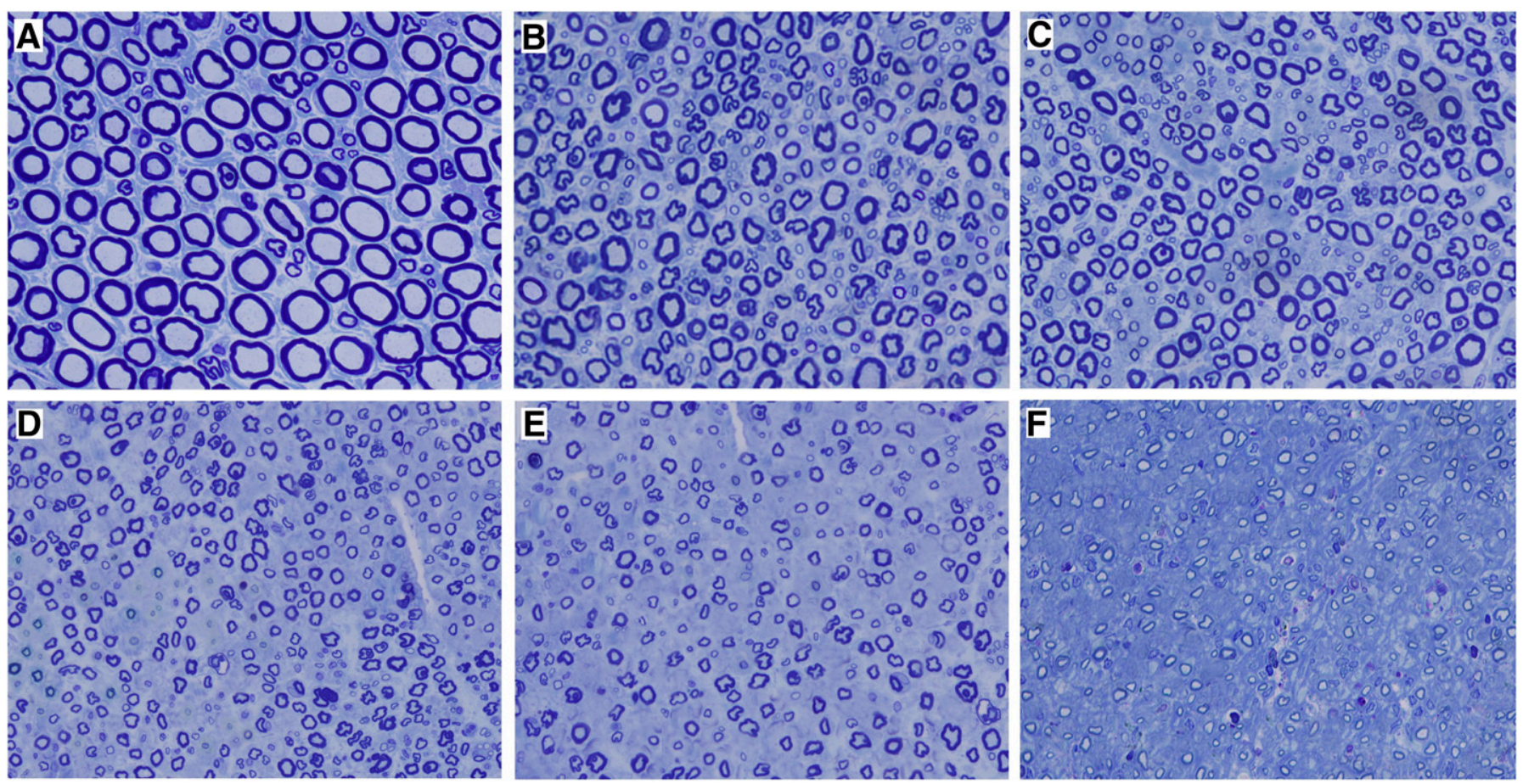

Fig. 10. Toluidine blue staining of semithin resin cross sections of distal sciatic nerve stumps at 16 weeks post-operation. Myelinated axons in control (A), autograft (B), ANA+ASCs+ALCAR (C), ANA+ALCAR (D), ANA+ASCs (E), and ANA alone (F). Scale bar, $50 \mu \mathrm{m}$.

well known that the efficacy of cell therapy will depend on the possibility of delivering a large number of viable functional cells into injured tissue (Potier et al., 2007). Therefore, we hypothesized that administration of ALCAR as a neuroprotective and antiapoptotic substance may be a way to increase survival rate and retention of transplanted ASCs under stressful conditions at the injury site. ALCAR is a short-chain ester of carnitine L-isomer that plays an important role in long-chain fatty acid transport across the mitochondrial membrane for $\beta$-oxidation and ATP production (McKay Hart et al., 2002; Bak et al., 2016). Also, it protects cells against lipid peroxidation and mitochondrial membrane breakdown through its antioxidative activity (Tesco et al., 1992; Gülçin, 2006; Zhang et al., 2012).

Therefore, we evaluated the effect of ALCAR treatment (50 $\mathrm{mg} / \mathrm{kg}$ per day) on the efficiency of acellular nerve graft for repair of a 10-mm gap in rat sciatic nerve. For this study, a dose of $50 \mathrm{mg} / \mathrm{kg}$ was chosen as the dose to provide a significant improvement in the nerve regeneration process (Wilson et al., 2010). For this purpose, we employed ANA, ANA supplemented with ASCs, and autograft as a control for reconstruction of a nerve defect. No signs of rejection and infection were observed. Our results showed that ANA provided a three-dimensional scaffold that supports axonal regeneration and muscle reinnervation. Results indicated that transplanted ASCs could significantly improve regenerative properties of acellular nerve graft in comparison with the acellular nerve graft group. But, their therapeutic capacity was still inferior to autograft group, which is in agreement with other studies (Scheib and Höke, 2013).

Therefore, ALCAR treatment led to improvement in the regenerative properties of ASC-supplemented ANA. ALCAR treatment led to significant improvement in locomotion recovery (as indexed by the SFI value) in ASC-supplemented ANA groups in comparison with untreated groups. However, no significant difference was observed in comparison with autograft. Also, results indicated that ALCAR treatment attenuated muscle mass loss during organ denervation. Electrophysiological assessment provided further evidence that nerve regeneration in the autograft group and ALCARtreated ASC-supplemented ANA groups was significantly superior to other groups. Furthermore, histologic evaluation showed that in the autograft group and ALCAR-treated

TABLE 2

Morphometric analyses of transverse sections at the sciatic nerve distal to injury for each of the experimental groups 16 weeks post-injury

Values are shown as mean \pm S.D.

\begin{tabular}{lccccc}
\hline \multicolumn{1}{c}{ Groups } & Axon Diameter & Myelin Thickness & Fiber Diameter & Myelinated Fiber Count & G-Ratio \\
\hline & $\mu m$ & $\mu m$ & $\mu m$ & & \\
Control & $0.31 \pm 4.92$ & $0.2 \pm 1.35$ & $0.68 \pm 7.63$ & $1053 \pm 7701$ & 0.64 \\
Autograft & $0.62 \pm 3.55$ & $0.2 \pm 1.11$ & $0.74 \pm 5.78$ & $2630 \pm 14,135$ & 0.61 \\
ANA & $0.39^{* * *} \pm 2.41$ & $0.16^{* * *} \pm 0.53$ & $0.7^{* * *} \pm 3.46$ & $2310^{* * * *} \pm 9271$ & 0.7 \\
ANA+ASCs & $0.58^{*} \pm 3.02$ & $0.18^{* * *} \pm 0.7$ & $0.89^{* * *} \pm 4.42$ & $1465^{* * * *} \pm 11,229$ & 0.68 \\
ANA+ALCAR & $0.57 \pm 3.1$ & $0.17^{* *} \pm 0.82$ & $0.22^{*} \pm 4.74$ & $1906 \pm 12,750$ & 0.65 \\
ANA+ASCs+ALCAR & $0.46 \pm 3.85$ & $0.23 \pm 1.04$ & $0.82 \pm 5.93$ & $1542^{*} \pm 16,064$ & 0.65 \\
\hline
\end{tabular}

$* P<0.05 ; * * P<0.01 ; * * * P<0.001$ vs. autograft group. 


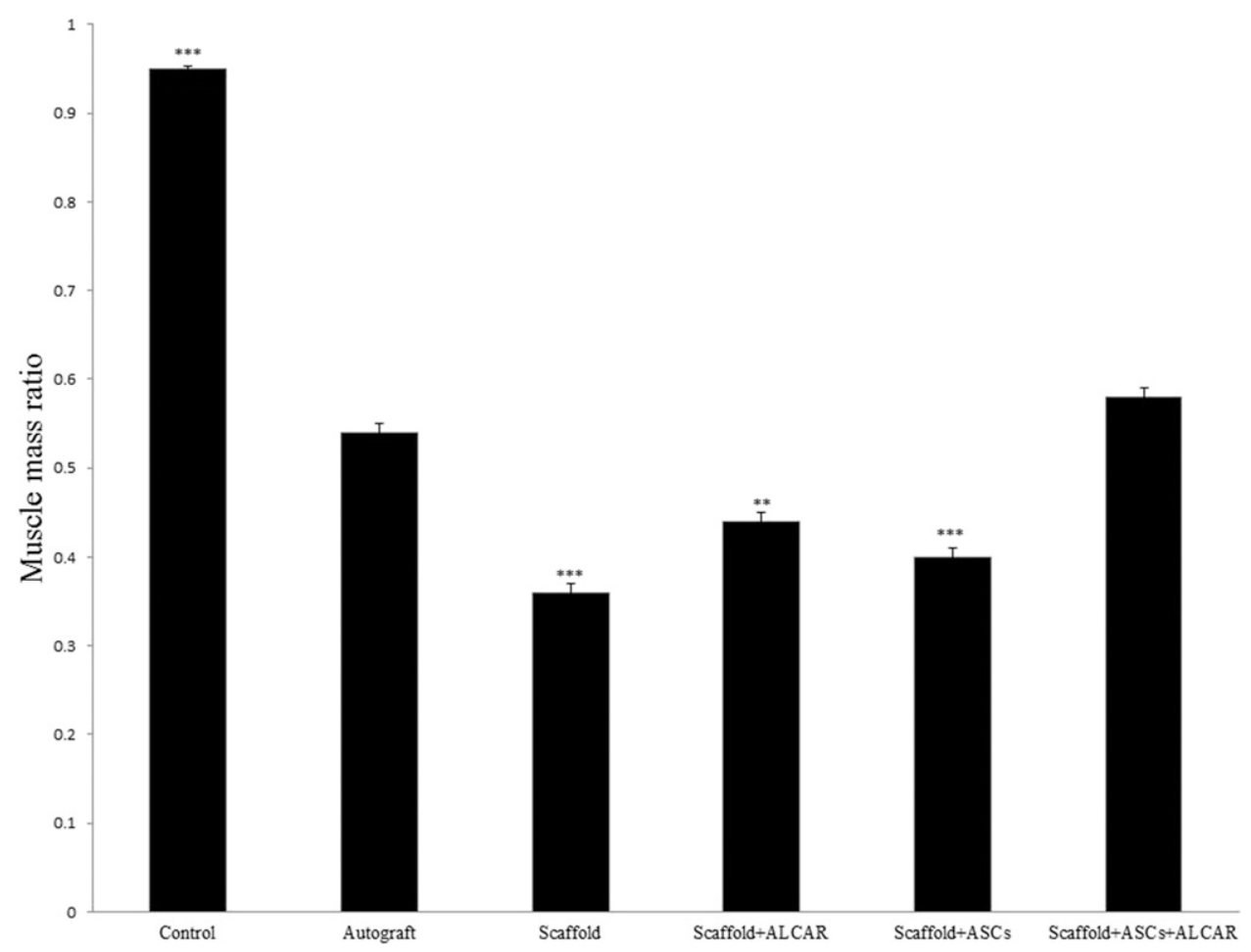

Fig. 11. Ratio measurement of gastrocnemius muscle weight. The gastrocnemius muscles of operated and unoperated sides were excised and weighed in the experimental groups at 16 weeks post-operative. Graph shows mean gastrocnemius muscle weight ratio (experimental/control). Data are shown as mean \pm S.D. $(n=10)$. $* * P<0.01 ; * * * P<0.001$ vs. autograft group.

allograft groups, more axons successfully crossed the graft from proximal to distal segments. ALCAR-treated allograft and autograft groups exhibited better reconstruction of regenerated nerve and of gastrocnemius target muscle than other groups. These data demonstrated that the systemic administration of ALCAR enhanced ASC-transplanted nerve allograft regenerative properties and resulting peripheral nerve regeneration.

The mechanism of the action of ASCs has not been definitively elucidated, but some studies suggest that they work by either releasing growth factors themselves or by modulating the endogenous Schwann cells (Lopatina et al., 2011). Regeneration of nerve injury depends on interaction between stromal cells and regenerative axons. The ability of stromal cells to secrete neurotrophic factors (such as NGF, GDNF, and $\mathrm{BDNF}$ ) plays an important role in successful neural recovery (Elgazzar et al., 2007).

Our results showed that the ALCAR-treated allografts supplemented with ASCs and the autograft group expressed stromal cell markers (S100, P75) and neurotrophic factors (NGF, GDNF, and BDNF). The mRNA levels of NGF and BDNF were similar between ALCAR-treated allografts supplemented with ASCs and the autograft group. Neurotrophins have been shown to improve neuronal survival and nerve regeneration in nerve injuries (Tang et al., 2013). The release of neurotrophins into acellular nerve grafts might contribute to the establishment of a native-like microenvironment for nerve regeneration in a manner similar to autografts. These factors may have partly originated from the ASCs that embedded in ANA or ASC-derived stromal cell-like cells. Some evidence has indicated that ASCs could differentiate into Schwann cell-like cells that express Schwann cell markers (Kingham et al., 2007). In vitro studies showed that differentiated ASCs enhance neurite outgrowth (Jiang et al., 2008) and this has been attributed to elevated levels of NGF and BDNF (Kingham et al., 2007). Therefore, we concluded that ALCAR treatment resulted in improve regenerative properties of cell-seeded nerve allograft probably by increasing the viability and retention of transplanted ASCs. However, the precise mechanism by which ALCAR improved nerve regeneration remains to be determined. It seems that ALCAR treatment could improve survival rate and retention of ASCs embedded in ANA and also improve peripheral nerve regeneration and functional recovery. Studies have shown that Schwann cell and ASC death occurs under ischemic conditions (Keilhoff et al., 2008). Some studies showed that ALCAR treatment has protective effect against stress-induced apoptosis in cultured cells (Ishii et al., 2000; Pillich et al., 2005). In agreement with these finding, our unpublished results showed that ALCAR (10 mM) treatment attenuated serumand glucose-deprivation-induced apoptosis in cultured rat ASCs. Likewise, Fujisawa and coworker confirmed that L-carnitine suppresses doxorubicin-induced apoptosis in bone marrow MSCs (Fujisawa et al., 2017). Other evidence indicated that ALCAR may prevent failure of mitochondrial oxidative metabolism (Bak et al., 2016; Altamimi et al., 2018). In vitro studies demonstrated that ALCAR was able to attenuate the rate of neuronal mortality (Manfridi et al., 1992). Also, in vivo studies indicated that ALCAR has a protective effect on peripheral nerve injuries (Fernandez et al., 1989; Hart et al., 2002). In this regard, it has been shown that ALCAR prevented sensory neuronal loss after axotomy by enhancing mitochondrial bioenergetics function (Hart et al., 2002). Some studies indicated that after nerve injury, ALCAR could attenuate mitochondrial oxidative stress and consequent mitochondrially induced cell death by reducing cytosolic levels of cytochrome $\mathrm{C}$ and caspase-3 active fragments (Di Cesare Mannelli et al., 2007). In accordance with these finding, our results showed that ALCAR treatment decreased caspase 3 mRNA expression at the site of nerve injury in ANA-ASC 
A



B

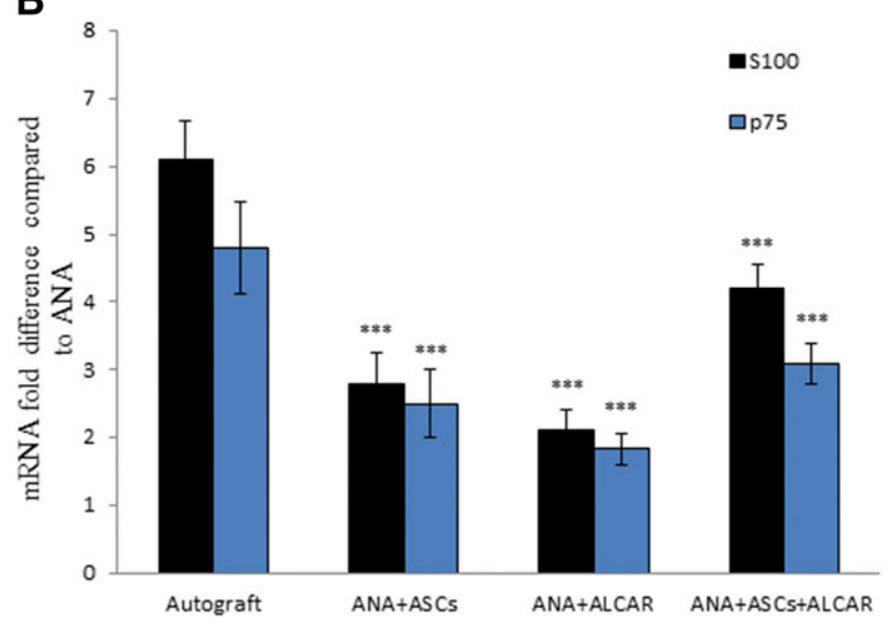

C

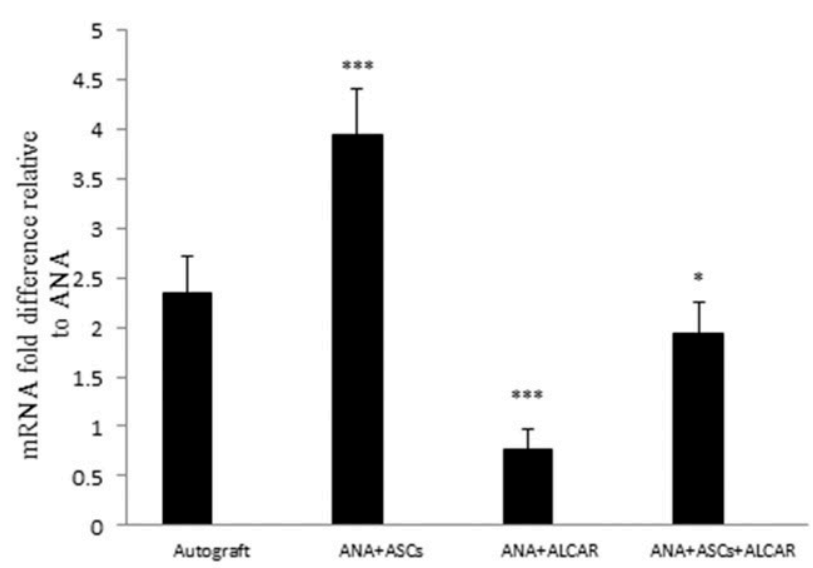

Fig. 12. Quantitative real-time PCR analyses of neurotrophin (A), Schwan cell markers (B), and caspase 3 (C) mRNA expression in implanted grafts. The mRNA level in naive rats has been ascribed a value of 1 and the vertical axis numbers indicate relative changes from this baseline for each group. The data are shown as mean \pm S.E.M. $(n=6) .{ }^{*} P<0.05,{ }^{*} P<0.01, * * * P<0.001 \mathrm{vs}$. autograft group.

construct-engrafted rat. Previous results showed that ALCAR treatment increase NGF responsiveness by enhancing the expression and affinity of neurotrophin receptors for NGF (Taglialatela et al., 1991; Manfridi et al., 1992).

Taken together, these findings suggest that ALCAR may be an effective neuroprotective agent for peripheral nerve regeneration that could improve the therapeutic efficacy of transplanted ASC-ANA constructs. In conclusion, our results showed that coapplication of acellular grafts incorporating ASCs as tissue-engineered ANA and ALCAR administration represent promising treatments for the repair of peripheral nerve large defects. This method can be a benefit that is easily adaptable to most clinical settings. Future studies should be done to confirm the precise mechanism of ALCAR function.

\section{Acknowledgments}

We thank Dr. Ann Paterson for assisting with English. The authors declare that they have no conflict of interest.

\section{Authorship Contributions}

Participated in research design: Ghayour, Behnam-Rassouli, Abdolmaleki. Conducted experiments: Ghayour, Abdolmaleki.

Performed data analysis: Mahdavi-Shahri Naser, Ghayour, Abdolmaleki.

Wrote or contributed to the writing of the manuscript: Ghayour, Abdolmaleki, Moghimi.

\section{References}

Altamimi TR, Thomas PD, Darwesh AM, Fillmore N, Mahmoud MU, Zhang L, Gupta A, Al Batran R, Seubert JM, and Lopaschuk GD (2018) Cytosolic carnitine acetyltransferase as a source of cytosolic acetyl-CoA: a possible mechanism for regulation of cardiac energy metabolism. Biochem $J$ 475:959-976.

Bak SW, Choi H, Park HH, Lee KY, Lee YJ, Yoon MY, and Koh SH (2016) Neuroprotective effects of acetyl-L-carnitine against oxygen-glucose deprivationinduced neural stem cell death. Mol Neurobiol 53:6644-6652.

Brohlin M, Mahay D, Novikov LN, Terenghi G, Wiberg M, Shawcross SG, and Novikova LN (2009) Characterisation of human mesenchymal stem cells following differentiation into Schwann cell-like cells. Neurosci Res 64:41-49.

Chavakis E, Urbich C, and Dimmeler S (2008) Homing and engraftment of progenitor cells: a prerequisite for cell therapy. $J \mathrm{Mol}$ Cell Cardiol 45:514-522.

Di Cesare Mannelli L, Ghelardini C, Calvani M, Nicolai R, Mosconi L, Vivoli E, Pacini A, and Bartolini A (2007) Protective effect of acetyl-L-carnitine on the apoptotic pathway of peripheral neuropathy. Eur $J$ Neurosci 26:820-827.

Dijkstra JR, Meek MF, Robinson PH, and Gramsbergen A (2000) Methods to evaluate functional nerve recovery in adult rats: walking track analysis, video analysis and the withdrawal reflex. J Neurosci Methods 96:89-96.

di Summa PG, Kalbermatten DF, Pralong E, Raffoul W, Kingham PJ, and Terengh G (2011) Long-term in vivo regeneration of peripheral nerves through bioengineered nerve grafts. Neuroscience 181:278-291.

Doolabh VB, Hertl MC, and Mackinnon SE (1996) The role of conduits in nerve repair: a review. Rev Neurosci 7:47-84.

Elgazzar R, Mutabgani M, Abdelaal S, and Sadakah A (2007) Platelet-rich plasma may enhance peripheral nerve regeneration after cyanoacrylate reanastomosis: a randomized blind study on rats. Int J Oral Maxillofac Surg 36:1031.

Fernandez E, Pallini R, Gangitano C, Del Fá A, Sangiacomo CO, Sbriccoli A, Ricoy JR, and Rossi GF (1989) Effects of L-carnitine, L-acetylcarnitine and gangliosides on the regeneration of the transected sciatic nerve in rats. Neurol Res 11:57-62.

Fujisawa K, Takami T, Fukui Y, Quintanilha LF, Matsumoto T, Yamamoto N, and Sakaida I (2017) Evaluating effects of L-carnitine on human bone-marrowderived mesenchymal stem cells. Cell and tissue research, $\mathbf{3 6 8}(2), 301-310$. 
Ghayour MB, Abdolmaleki A, and Behnam-Rassouli M (2017) The effect of Riluzole on functional recovery of locomotion in the rat sciatic nerve crush model. Eur J Trauma Emerg Surg 43:691-699.

Gimble JM, Katz AJ, and Bunnell BA (2007) Adipose-derived stem cells for regenerative medicine. Circ Res 100:1249-1260.

Gülçin I (2006) Antioxidant and antiradical activities of L-carnitine. Life Sci 78: 803-811.

Hargreaves K, Dubner R, Brown F, Flores C, and Joris J (1988) A new and sensitive method for measuring thermal nociception in cutaneous hyperalgesia. Pain 32 77-88.

Hart AM, Wiberg M, Youle M, and Terenghi G (2002) Systemic acetyl-L-carnitine eliminates sensory neuronal loss after peripheral axotomy: a new clinical approach in the management of peripheral nerve trauma. Exp Brain Res 145:182-189.

Hea Gu J, Hwa Ji Y, Dhong ES, Hwee Kim D, and Yoon ES (2012) Transplantation of adipose derived stem cells for peripheral nerve regeneration in sciatic nerve defects of the rat. Curr Stem Cell Res Ther 7:347-355.

Ishii T, Shimpo Y, Matsuoka Y, and Kinoshita K (2000) Anti-apoptotic effect of acetyll-carnitine and I-carnitine in primary cultured neurons. Jpn J Pharmacol 83 $119-124$.

Jessen KR and Mirsky R (2008) Negative regulation of myelination: relevance for development, injury, and demyelinating disease. Glia 56:1552-1565.

Jiang L, Zhu JK, Liu XL, Xiang P, Hu J, and Yu WH (2008) Differentiation of rat adipose tissue-derived stem cells into Schwann-like cells in vitro. Neuroreport 19 $1015-1019$

Keilhoff G, Schild L, and Fansa H (2008) Minocycline protects Schwann cells from ischemia-like injury and promotes axonal outgrowth in bioartificial nerve grafts lacking Wallerian degeneration. Exp Neurol 212:189-200.

Kingham PJ, Kalbermatten DF, Mahay D, Armstrong SJ, Wiberg M, and Terenghi G (2007) Adipose-derived stem cells differentiate into a Schwann cell phenotype and promote neurite outgrowth in vitro. Exp Neurol 207:267-274.

Kokai LE, Rubin JP, and Marra KG (2005) The potential of adipose-derived adult stem cells as a source of neuronal progenitor cells. Plast Reconstr Surg 116 1453-1460.

Kuroda Y, Kitada M, Wakao S, Nishikawa K, Tanimura Y, Makinoshima H, Goda M, Akashi H, Inutsuka A, Niwa A, et al. (2010) Unique multipotent cells in adult human mesenchymal cell populations. Proc Natl Acad Sci USA 107:8639-8643.

Liu G, Cheng Y, Guo S, Feng Y, Li Q, Jia H, Wang Y, Tong L, and Tong X (2011) Transplantation of adipose-derived stem cells for peripheral nerve repair. Int $\mathrm{J} \mathrm{Mol}$ Med 28:565-572.

Locke M, Windsor J, and Dunbar PR (2009) Human adipose-derived stem cells: isolation, characterization and applications in surgery. ANZ J Surg 79:235-244

Lopatina T, Kalinina N, Karagyaur M, Stambolsky D, Rubina K, Revischin A, Pavlova G, Parfyonova Y, and Tkachuk V (2011) Adipose-derived stem cells stimulate regeneration of peripheral nerves: BDNF secreted by these cells promotes nerve healing and axon growth de novo. PLoS One 6:e17899.

Lu Q, Zhang Y, and Elisseeff JH (2015) Carnitine and acetylcarnitine modulate mesenchymal differentiation of adult stem cells. J Tissue Eng Regen Med $\mathbf{9}$ $1352-1362$.

Mackinnon SE and Hudson AR (1992) Clinical application of peripheral nerve transplantation. Plast Reconstr Surg 90:695-699.

Manfridi A, Forloni GL, Arrigoni-Martelli E, and Mancia M (1992) Culture of dorsa root ganglion neurons from aged rats: effects of acetyl-L-carnitine and NGF. Int $J$ Dev Neurosci 10:321-329.

Mansour HH (2006) Protective role of carnitine ester against radiation-induced oxidative stress in rats. Pharmacol Res 54:165-171.

McKay Hart A, Wiberg M, and Terenghi G (2002) Pharmacological enhancement of peripheral nerve regeneration in the rat by systemic acetyl-L-carnitine treatment. Neurosci Lett 334:181-185.
McKenzie JL, Gan OI, Doedens M, Wang JC, and Dick JE (2006) Individual stem cells with highly variable proliferation and self-renewal properties comprise the human hematopoietic stem cell compartment. Nat Immunol 7:1225-1233.

Mosahebi A, Fuller P, Wiberg M, and Terenghi G (2002) Effect of allogeneic Schwann cell transplantation on peripheral nerve regeneration. Exp Neurol 173:213-223.

Navarro X and Udina E (2009) Chapter 6: methods and protocols in peripheral nerve regeneration experimental research: part III-electrophysiological evaluation. Int Rev Neurobiol 87:105-126.

Neubauer D, Graham JB, and Muir D (2010) Nerve grafts with various sensory and motor fiber compositions are equally effective for the repair of a mixed nerve defect. Exp Neurol 223:203-206.

Oğuzhanoğlu A, Erdoğan C, Tabak E, and Cenikli U (2010) Comparison of conduction velocities of nerve fibers to smaller and larger muscles in rats. Int $J$ Neurosci 120: $76-79$

Pillich RT, Scarsella G, and Risuleo G (2005) Reduction of apoptosis through the mitochondrial pathway by the administration of acetyl-L-carnitine to mouse fibroblasts in culture. Exp Cell Res 306:1-8.

Potier E, Ferreira E, Meunier A, Sedel L, Logeart-Avramoglou D, and Petite H (2007) Prolonged hypoxia concomitant with serum deprivation induces massive human mesenchymal stem cell death. Tissue Eng 13:1325-1331.

Raimondo S, Fornaro M, Di Scipio F, Ronchi G, Giacobini-Robecchi MG, and Geuna S (2009) Chapter 5: methods and protocols in peripheral nerve regeneration experimental research: part II-morphological techniques. Int Rev Neurobiol 87:81-103.

Scheib J and Höke A (2013) Advances in peripheral nerve regeneration. Nat Rev Neurol 9:668-676.

Siemionow M, Bozkurt M, and Zor F (2010) Regeneration and repair of peripheral nerves with different biomaterials: review. Microsurgery 30:574-588.

Sondell M, Lundborg G, and Kanje M (1998) Regeneration of the rat sciatic nerve into allografts made acellular through chemical extraction. Brain Res 795:44-54.

Taglialatela G, Angelucci L, Ramacci MT, Werrbach-Perez K, Jackson GR, and Perez-Polo JR (1991) Acetyl-L-carnitine enhances the response of PC12 cells to nerve growth factor. Brain Res Dev Brain Res 59:221-230.

Tang S, Zhu J, Xu Y, Xiang AP, Jiang MH, and Quan D (2013) The effects of gradients of nerve growth factor immobilized PCLA scaffolds on neurite outgrowth in vitro and peripheral nerve regeneration in rats. Biomaterials 34:7086-7096.

Tesco G, Latorraca S, Piersanti P, Piacentini S, Amaducci L, and Sorbi S (1992) Protection from oxygen radical damage in human diploid fibroblasts by acetyl-Lcarnitine. Dement Geriatr Cogn Disord 3:58-60.

Tohill M and Terenghi G (2004) Stem-cell plasticity and therapy for injuries of the peripheral nervous system. Biotechnol Appl Biochem 40:17-24.

Toma C, Pittenger MF, Cahill KS, Byrne BJ, and Kessler PD (2002) Human mesenchymal stem cells differentiate to a cardiomyocyte phenotype in the adult murine heart. Circulation 105:93-98.

Wilson AD, Hart A, Wiberg M, and Terenghi G (2010) Acetyl-l-carnitine increases nerve regeneration and target organ reinnervation - a morphological study. J Plast Reconstr Aesthet Surg 63:1186-1195.

Zhang R, Zhang H, Zhang Z, Wang T, Niu J, Cui D, and Xu S (2012) Neuroprotective effects of pre-treatment with l-carnitine and acetyl-L-carnitine on ischemic injury in vivo and in vitro. Int $J$ Mol Sci 13:2078-2090.

Zhang Y, Luo H, Zhang Z, Lu Y, Huang X, Yang L, Xu J, Yang W, Fan X, Du B, et al. (2010) A nerve graft constructed with xenogeneic acellular nerve matrix and autologous adipose-derived mesenchymal stem cells. Biomaterials 31:5312-5324.

$\overline{\text { Address correspondence to: Dr. Morteza Behnam-Rassouli, Department of }}$ Biology, Ferdowsi University, Azadi Square, Mashhad 9177948974, Iran. E-mail: ari_1364@yahoo.com 